\title{
Consideration of various Noise Types and Illumination Effects for 3D shape recovery
}

\author{
Aamir Saeed Malik and Tae-Sun Choi \\ Gwangju Institute of Science and Technology \\ Republic of Korea
}

\section{Introduction}

There are a variety of 3D Shape estimation methods. Broadly these methods can be classified into three types, namely, Contact, Transmissive and Reflective methods. The "Contact" method is generally based on some physical contact to acquire data while the "Transmissive" method is based on sending waves (like electromagnetic radiations, sound waves etc) through a body and recording data because of the interaction of wave particles with the object under consideration. The reflective model acquires data based on reflection of wave particles. The reflective method is broadly divided into optical and non-optical techniques.

The optical methods under the reflective model can further be divided into "Passive" and "Active" Techniques. In active techniques, we are projecting light rays while in passive techniques; we simply capture the reflection of light rays without any projections. The passive methods can further be classified as Shape From X (Stereo, Motion, Shading, Focus etc). This chapter deals with Shape From Focus (SFF) which is a passive optical method. The objective of shape from focus is to find out the depth of every point of the object from the camera lens. Hence, we obtain a depth map which contains the depth of all points of the object from the camera lens where they are best focused.

The aim of this chapter is to study the various factors (for example, different types of noise, illumination, window size) that affect SFF. It is shown that the illumination effects can directly result in incorrect estimation of depth map if proper window size is not selected during the computation of focus measure. The large window size results in blurring the image which gives the wrong impression of smoothness of the depth map. So it is important to find the optimum window size for accurate depth map estimation. Further, it is shown that the images need some kind of pre-processing to enhance the dark regions and shadows in the image.

Additionally, a robust focus measure is also discussed in this chapter. This focus measure has shown robustness in the presence of noise as compared to the earlier focus measures. This new focus measure is based on an optical transfer function implemented in the Fourier domain. The focus measure is tested at various levels of noise, i.e., low, medium and high noise levels. The results of this focus measure have shown drastic improvement in estimation of depth map, with respect to the earlier focus measures, in the presence of various types of noise including Gaussian, Shot and Speckle noise. 


\section{Shape From Focus (SFF)}

The basic problem of imaging systems, such as the eye or a video-camera, is that depth information is lost while projecting a 3D scene onto 2D image plane. Therefore, one of the fundamental problem in computer vision is the reconstruction of a geometric object from one or several observations. Various image processing techniques retrieve the lost cue and shape information from the pictorial information. Shape from focus (Krotkov, 1987) is one of such image processing techniques that are used to recover 3D information.

The basic image formation geometry is shown in Figure 1. In the figure, the parameters related to the camera are already known. We need to calculate ' $u$ ', i.e., depth of object from the lens. We make a depth map by calculating ' $u$ ' for every pixel. We can use the lens formula to calculate ' $u$ '. If the image detector (ID) is placed exactly at a distance $v$, sharp image $\mathrm{P}^{\prime}$ of the point $\mathrm{P}$ is formed. Then the relationship between the object distance $\mathrm{u}$, focal distance of the lens $f$, and the image distance $v$ is given by the Gaussian lens law:

$$
\frac{1}{f}=\frac{1}{u}+\frac{1}{v}
$$

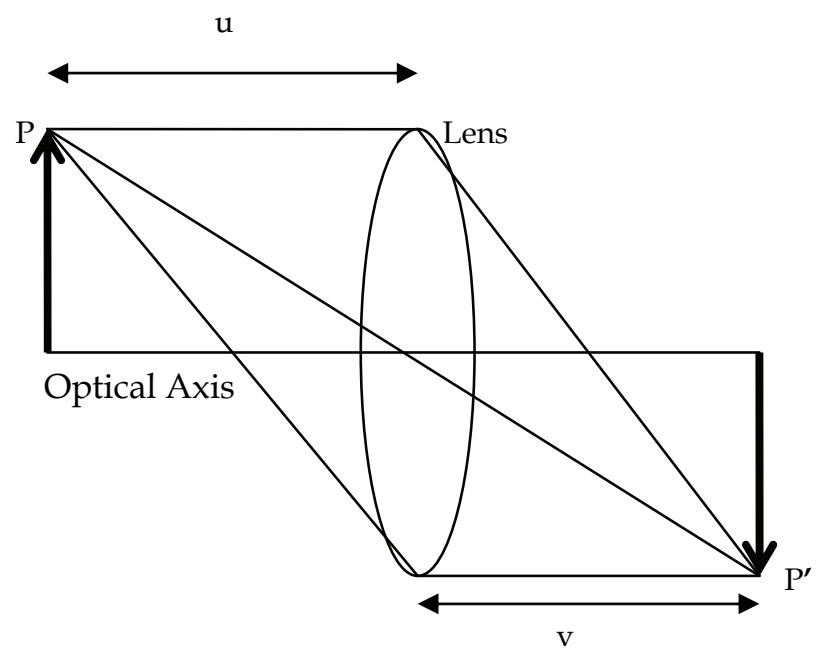

Figure 1. Image Formation of a 3D Object

Therefore, in SFF, a sequence of images that correspond to different levels of object focus is obtained. A sharp image and the relative depth can be retrieved by collecting the best focused points in each image. The absolute depth of object surface patches can be calculated from the focal length and the position of lens that give the sharpest image of the surface patches. The depth or best focus is thus obtained by using some focus measure.

One factor is to be kept in mind that we have finite number of images in the image sequence. The information obtained from them does not represent actual object specification especially in the case of geometrically complex objects. The only way for obtaining accurate results from SFF techniques is estimating object specifications in the gap between images in 
the image sequence. Hence, the role of approximation techniques is very important after getting the initial result from focus measure.

The objective of shape from focus is to find out the depth of every point of the object from the camera lens. Hence, finally we get a depth map which contains the depth of all points of the object from the camera lens where they are best focused or in other words, where they show maximum sharpness. Therefore, in SFF, a sequence of images that correspond to different levels of object focus is obtained.

To measure the true focussed point requires large number of images with incremental distance moved towards focus plane. To detect the true focussed point from finite number of images, various focus measures have been proposed by researchers. A focus measure is a quantity which measures the degree of blurring of an image; its value is a maximum when the image is best focused and decreases as blurring increases. Figure 2 shows a focus measure curve for a point in the image.

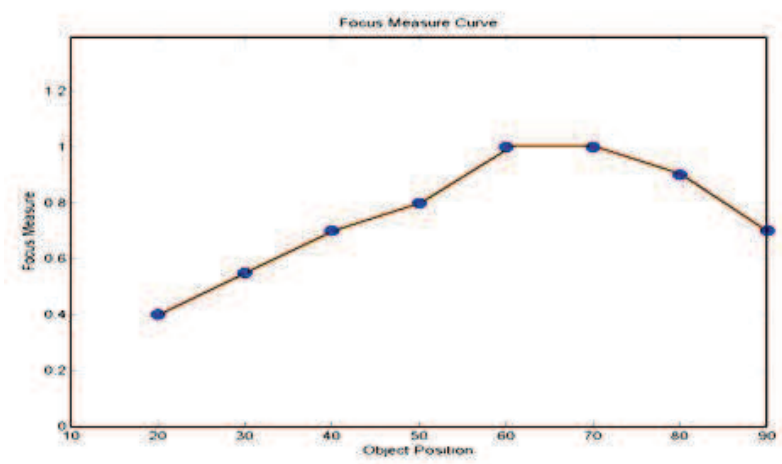

Figure 2. Focus Measure Curve for a point

\section{Related Work}

\subsection{Focus Measure}

A Focus Measure operator is one that calculates the best focused point in the image, i.e., focus measure is defined as a quantity to evaluate the sharpness of a pixel locally. The value of the focus measure increases as the image sharpness increases and attains the maximum for the best focused image. (Helmli \& Scherer, 2001) summarized the traditional focus measures while introducing new focus measure operators. Existing focus measure operators are given in brief below:

Sum of Modified Laplacian (SML): If the image has rich textures with high variability at each pixel, focus measure can be calculated considering single pixel. In order to improve robustness for weak-texture images, (Nayar \& Nakagawa, 1994) presented focus measure at $(x, y)$ as Sum of Modified Laplacian values in a local window (about $5 x 5)$ around $(x, y)$.

$$
\operatorname{SML}\left(x_{0}, y_{0}\right)=\sum_{p(x, y) \in U\left(x_{0}, y_{0}\right)}\left(\frac{\partial^{2} g(x, y)}{\partial^{2} x}\right)^{2}+\left(\frac{\partial^{2} g(x, y)}{\partial^{2} y}\right)^{2}
$$


Tenenbaum Focus Measure $\left(\mathbf{F M}_{\mathrm{T}}\right)$ : It is gradient magnitude maximization method that measures the sum of squared responses of horizontal and vertical Sobel masks. For robustness, it is also summed in a local window.

$$
F M_{T}\left(x_{0}, y_{0}\right)=\sum_{p(x, y) \in U\left(x_{0}, y_{0}\right)}\left(G_{x}(x, y)^{2}+G_{y}(x, y)^{2}\right)^{2}
$$

Gray Level Variance (GLV) Focus Measure: In case of a sharp image, the variance of graylevel is higher than that in a blur image.

$$
G L V\left(x_{0}, y_{0}\right)=\frac{1}{N-1} \sum_{p(x, y) \in U\left(x_{0}, y_{0}\right)}\left(g(x, y)-\mu_{U\left(x_{0}, y_{0}\right)}\right)^{2}
$$

With $\mu_{U\left(x_{0}, y_{0}\right)}$ the mean of the gray values in the neighborhood $\mathrm{U}\left(\mathrm{x}_{0}, \mathrm{y}_{0}\right)$

Mean Method Focus Measure $\left(\mathbf{F M}_{\mathbf{M}}\right)$ : The ratio of mean grey value to the center grey value in the neighborhood can also be used as a focus measure. The ratio of one shows a constant grey-level or absence of texture. The ratio is different in case of high variations. It is also summed in a local window. Let FM' is the ratio of mean grey value to the center grey value:

$$
F M_{M}\left(x_{0}, y_{0}\right)=\sum_{p(x, y) \in U\left(x_{0}, y_{0}\right)} F M^{\prime}(x, y)
$$

Curvature Focus Measure (FMC): The curvature in a sharp image is expected to be higher than that in a blur image. First, the surface is approximated using a quadratic equation $f(x, y)$ $=a x+b y+c x^{2}+d y^{2}$. The coefficients $(a, b, c, d)$ are calculated using a least squares approximation technique. Then these coefficients are combined to obtain a focus measure.

$$
\operatorname{FM}_{\mathrm{c}}(\mathrm{x}, \mathrm{y})=|\mathrm{a}|+|\mathrm{b}|+|\mathrm{c}|+|\mathrm{d}|
$$

$\mathbf{M}_{2}$ Focus Measure: Various focus measures were proposed by (Subbarao et al., 1993). The focus measures proposed were based on image grey level variance $\left(M_{1}\right)$, energy of image gradient $\left(\mathrm{M}_{2}\right)$ and energy of image Laplacian $\left(\mathrm{M}_{3}\right)$. These focus measures are similar to those described above, i.e., $M_{1}$ is similar to Gray Level Variance (GLV) Focus Measure, $M_{2}$ is similar to Tenenbaum Focus Measure, and $\mathrm{M}_{3}$ is similar to Laplacian focus measure. $\mathrm{M}_{2}$ is computed as:

$$
\mathrm{M}_{2}=\sum_{x=i-N}^{i+N} \sum_{y=j-N}^{j+N}\left(\mathrm{~g}_{\mathrm{x}}^{2}+\mathrm{g}_{\mathrm{y}}^{2}\right)
$$

where: $g_{x}(x, y)=g_{i}(x+1, y)-g_{i}(x, y) \& g_{y}(x, y)=g_{i}(x, y+1)-g_{i}(x, y)$

\subsection{Approximation Methods}

The discrete number of frames results in some loss of information in between frames. As a result, the optimum value for some pixels may never be calculated. Hence to address this issue among others, approximation techniques can be applied to the results of the focus measures to construct a more accurate depth range image. (Malik \& Choi, 2007) summarized 
various approximation techniques. They found that in Traditional (TR) SFF, for each image in the sequence the Focus Measure at each pixel can be computed by the Sum Modified Laplacian in the 2D neighborhood around the pixel. Thus, for each pixel, the image frame with the maximum Focus Measure is determined. The camera parameters for this image frame are then used to compute the distance of the object point corresponding to that pixel.

It should be noted that these traditional methods do not consider the fact that an image of 3D object is also three dimensional in image space. Therefore, (Subbarao \& Choi, 1995) proposed a new concept they refer to as Focused Image Surface (FIS) applied on SML, which is based on planar surface approximations. The FIS of an object is defined as the surface formed by the set of points at which the object points are focused by a camera lens, after first obtaining an estimate of FIS using a traditional SFF method. This estimate is then refined by searching for a planar surface that maximizes the Focus Measure computed over pixels on FIS. (Choi et al., 1999) proposed the approximation of FIS by a piecewise curved surface rather than through the use of a piecewise planar approximation, where the piecewise curved surface is estimated by interpolation using a second order Lagrange polynomial.

(Asif \& Choi, 2001) used Neural Networks on GLV result to learn the shape of FIS by optimizing the Focus Measure over small 3D windows, as due to their nonlinear characteristics, neural networks can be used to approximate any arbitrary function. (Bilal \& Choi, 2005) proposed the use of Dynamic Programming (DP) on SML result for handling the computational complexity of FIS. Based on DP definition, a large problem can be split into a series of smaller problems. Thus, unlike the FIS approach, DP can search for the optimal Focus Measure in the whole image volume rather than being limited to a small neighborhood. However, the direct application of DP on 3D data is impractical due to its computational complexity; consequently, they proposed a heuristic model based on DP.

\section{Illumination and Window Size}

In this section, the main emphasis is on the illumination problems and the corresponding window size affecting the images. The reason for selection of this factor, i.e., illumination, is that almost all the images are affected by illumination. Proper illumination is only possible in well controlled lab conditions. But in real time imaging, it's not possible. Hence, the images have regions with diverse illuminations. However, the regions with low illumination are the ones that require special attention while estimating the depth maps because such regions result in the selection of incorrect focused points for the depth map.

The previous work regarding estimation of depth map using SFF has been discussed in section 3. Here, only the effect of the window sizes are discussed with respect to those methods. In the literature, the trend is to use large window size, e.g., window size of $11 \times 11$ has been used commonly to compute various focus measures including Tenenbaum, Gray Level Variance (GLV), Mean Method, Curvature and Point focus measures. (Ahmad \& Choi, 2005) mentioned using 15x15 window size for computation of TR SFF. (Subbarao \& Choi, 1995) used energy of Laplacian as the focus measure and used window of size 15x15 to compute the focus measure to implement the Focused Image Surface method. For initial estimate, (Choi et al., 1999) used Sum of Modified Laplacian as the focus measure for curved window technique and used window of size 15x15. (Asif \& Choi, 2001) used Gray Level Variance (GLV) as the focus measure for their Neural Network based method with window of size 7x7. (Ahmad \& Choi, 2005) used Sum of Modified Laplacian as the focus measure for the dynamic programming based method and used window of size $7 \times 7$. 
Here, we show the results with one of the focus measure, i.e., Gray Level Variance (GLV). We acquired a sequence of 97 real cone images, each at different focus value. Figure 3 shows three fames of the real cone. It is evident from the images that maximum illumination occurs at the upper side of the images hence, the upper part or one side of the cone is quite bright. Then as we move down the image vertically, the illumination decreases but still the sides of the cones are well illuminated. Finally at the bottom of the image, i.e., the region of the image right below the tip of the cone extending till end of the cone is quite dark. Hence three distinct regions of illumination can easily be identified from these images.

Figure 4 shows the images when the GLV focus measure is applied to various frames of the real cone images. The images shown in first column are computed using the window size of $3 \times 3$, the ones in second column are computed using window size of $5 \times 5$ and window size of $7 \times 7$ is used for those in third column. It can be seen from the figure that the parts below the tip of the cone are not well focused because of poor illumination. However, with the increase in the window size, the number of pixels being extracted increase in the low illumination region.

From figure 4 , it is clear that the blurring effect is more pronounced in the $3^{\text {rd }}$ column $(7 \times 7$ window size) as compared to first column ( $3 \times 3$ window size). The number of pixels extracted has increased in the $3^{\text {rd }}$ column because of consideration of more neighborhood pixels with dissimilar values. This clearly indicates that the dependence on sharpness of the pixel value itself has decreased while the dependence on the values of the neighborhood pixels has increased. So, the larger the window size, the more is probability of taking into account higher pixel values of the neighbouring pixels lying far from the pixel in consideration. Hence, the result will be incorrect selection of frame numbers (that may not correspond to the best focus point) during computation of the depth map. We emphasize on this fact because people have used large window size like 7x7, 11x11 and 15x15 etc in the literature as discussed earlier. Although, we can show more results with other focus measures too but the above mentioned two points continue to hold.
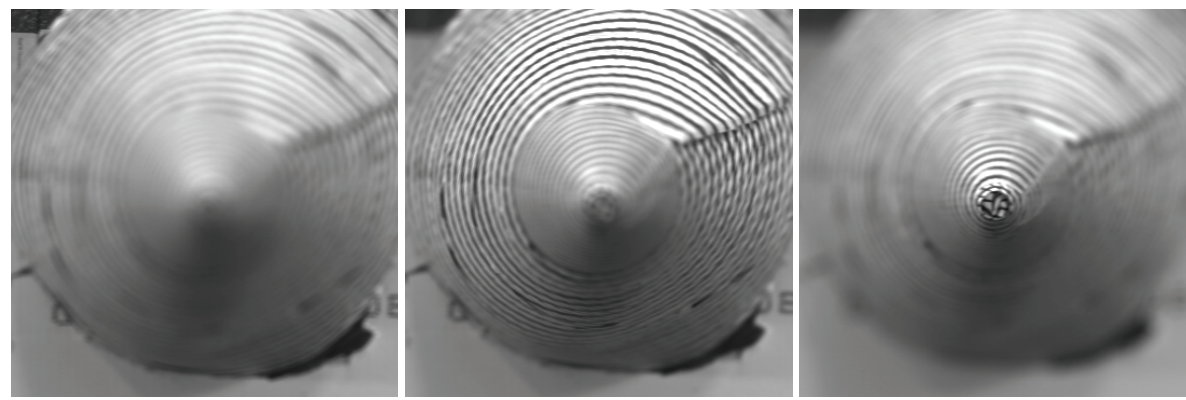

Figure 3. Various frames of real cone at different focus values

Now consider figure 5. We present the results of depth map from Sum of Modified Laplacian using window size of $7 \times 7,9 \times 9$ and $11 \times 11$. This figure clearly shows the effects of increasing the size of windows. As the size of the window increase, the result is smoothing and hence we get much smoother depth maps compared to those that are obtained by using smaller window size. This smoothing is because of the blurring effect that is introduced due to large window size. The larger window size takes into account more pixel values which 
might be quite different from the pixel in consideration. Hence, as the number of neighboring pixels increases, the worth of local intensity variation reduces.
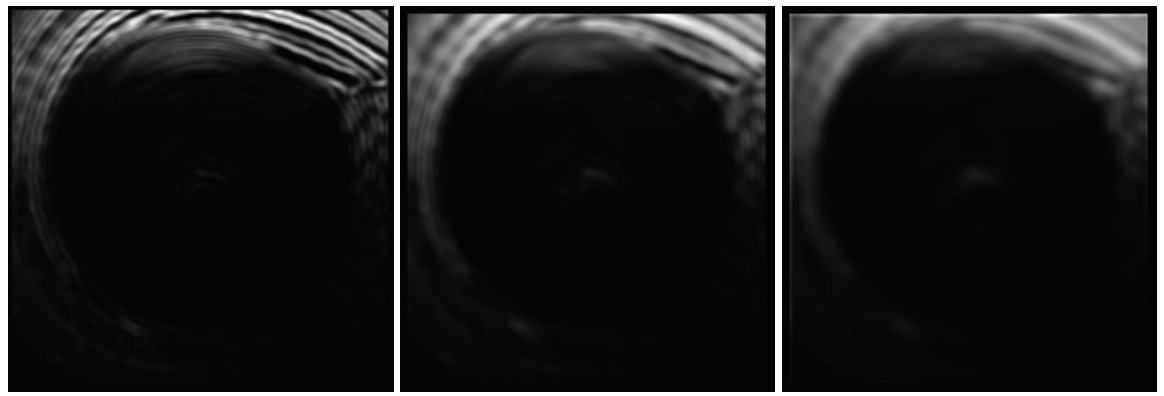

(a) Frame 50
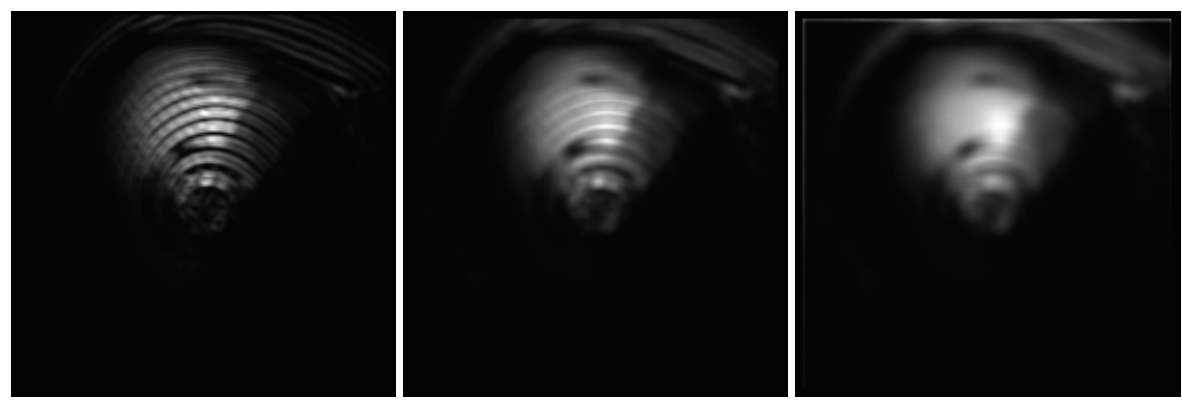

(b) Frame 75
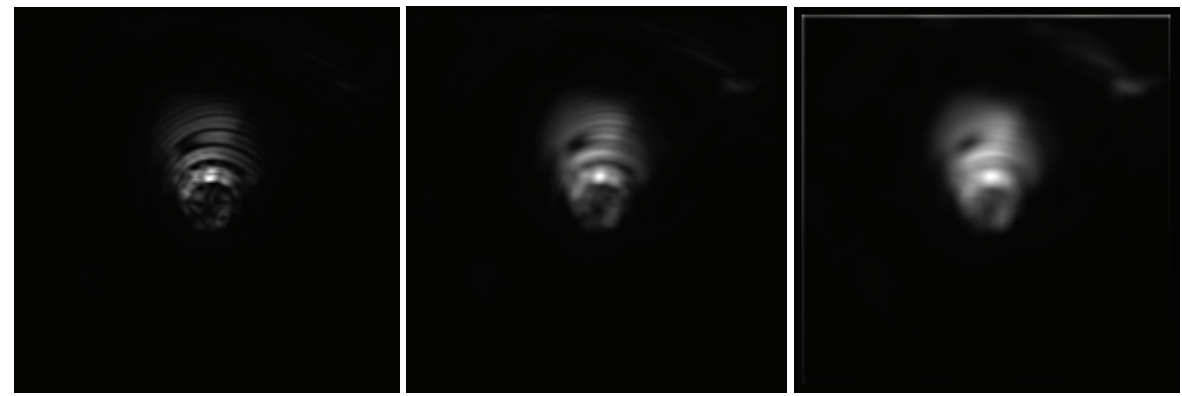

(c) Frame 90

Figure 4. Gray Level Variance operation performed with different window sizes

Also, it can be seen from the images that as the size of the window increases, the number of pixels extracted also increase. Again this is because more neighborhood pixels are taken into account. Those neighborhood pixels might lie in well illuminated region compared to the pixel in consideration that was not extracted earlier. Hence, this results in incorrect estimation of depth map because the dependence is now on the values of the neighbors that lie in or near the high or adequate illuminated region.

So, from all this discussion, we safely conclude that if some parts of the object in an image lie in the region of low illumination then that part of the object cannot be extracted by 
directly applying the edge extraction methods or techniques that find the sharp points in the images. In such cases, the images need to be pre-processed so that low illumination regions can be enhanced. Further, the larger the window size, the more is probability of taking into account higher pixel values of the neighbouring pixels lying far from the pixel in consideration. Hence, the depth map will contain incorrect frame numbers pointing to the wrong pixel values. So, a smaller window size should be used for computation of focus measures. Window size of $3 \times 3$ is adequate for such computations. However, the upper bound or the upper limit on window size should be $5 \times 5$. Any selection of window size greater than $5 \times 5$ will introduce errors in the depth map estimation (Malik \& Choi, 2007).

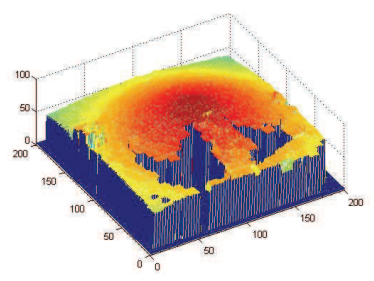

(a) $7 \times 7$

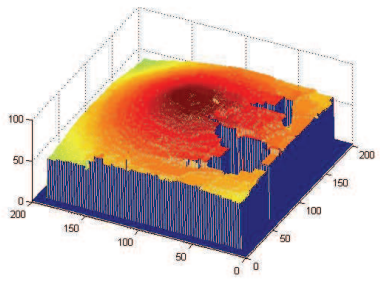

(b) $9 \times 9$

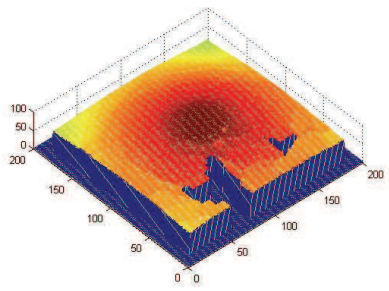

(c) $11 \times 11$

Figure 5. Depth Maps computed from Sum of Modified Laplacian using various window sizes

\section{Noise Impact on SFF}

As discussed earlier in section 3, there are various focus measures available to estimate the depth map. Three of them are based on second derivative. They are namely Laplacian, Modified Laplacian and Sum of Modified Laplacian. But the problem with second derivative is that it is extremely sensitive to noise. Hence, the result is degraded drastically if there is noise in the image. Another focus measure, Tenenbaum Focus Measure, is based on single derivative technique which again is sensitive to noise although its less sensitive compared to double derivative techniques. Same problem is observed with focus measures incorporating mean and gray level variance values. The pixels, with noise addition, are enhanced and hence taken as sharp focused points when variance values are taken into account. In an extension of variance of gray level method, another method is mean method focus measure. But this technique also suffers from the same problem like gray level variance method.

In this section, three different types of noise are considered, i.e., Gaussian, Speckle and Shot noise. Gaussian noise is used to model the thermal noise which is due to the additional electrons generated within the CCD by physical processes within the CCD itself. Shot noise is found in situations where quick transients, such as faulty switching, take place during imaging. Speckle noise is a physical effect, which occurs when coherent light is reflected from an optically rough surface. Two objects, simulated cone and the real cone, are used and their depth maps are estimated in the presence of these noise types. Now consider figure 6(a). One of the frames of the original cone image is shown without noise. Figure 6(b) shows the same frame when Gaussian noise (mean=0, variance $=0.005)$ is added. Figure 6(c) shows 
the result of Laplacian operator and it is quite clear that the Laplacian processed result has been degraded significantly.

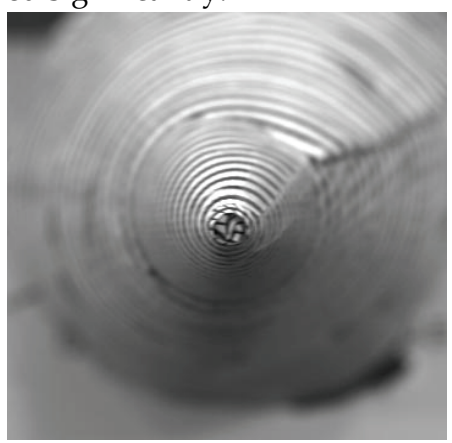

(a) Original

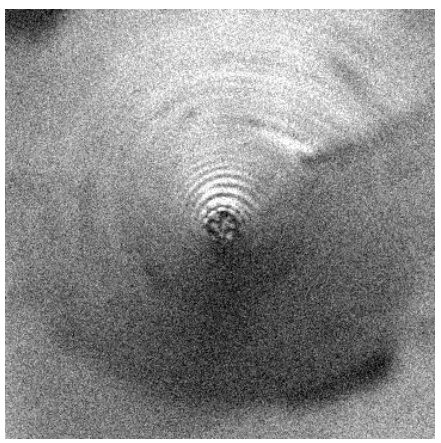

(b) With Gaussian Noise

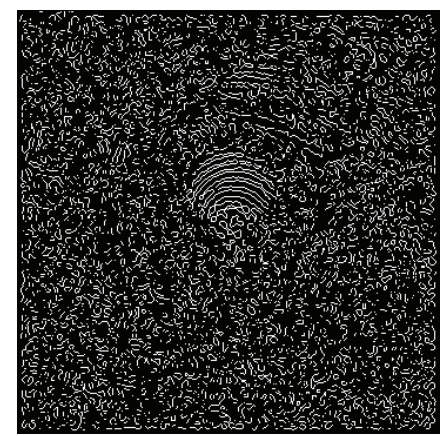

(c) Laplacian

Figure 6. Effect of adding Gaussian noise

Figure 7 shows the depth maps obtained using SML focus measure for simulated cone images. The depth map on the left hand side shows the depth map obtained when no noise is added to the images. The depth map on the right hand side shows the depth map when Gaussian noise (mean $=0$, variance $=0.005$ ) is added to the images. It is evident from these depth maps that the results degrade significantly in the presence of noise.

Figure 8 shows the depth maps obtained using SML focus measure for real cone images. However, this time shot noise is added in figure $8(\mathrm{~b})$ and speckle noise in figure 8(c). It is again evident from these depth maps that the results degrade significantly in the presence of shot and speckle noise. The noise has enhanced the individual pixel values and hence resulted in spikes all over the depth map. With this result, it is not possible to further refine it using some approximation method as discussed in section 3. We have analyzed various focus measures and found that all of the focus measures are effected when noise is present in the images. 


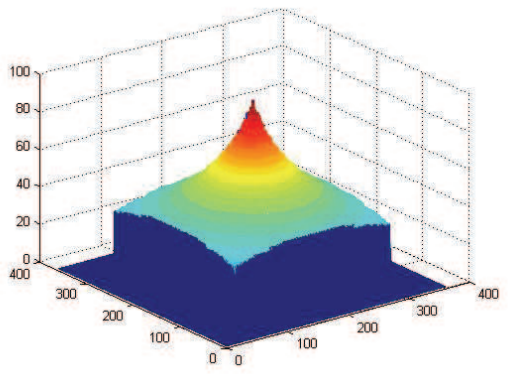

(a) No Noise

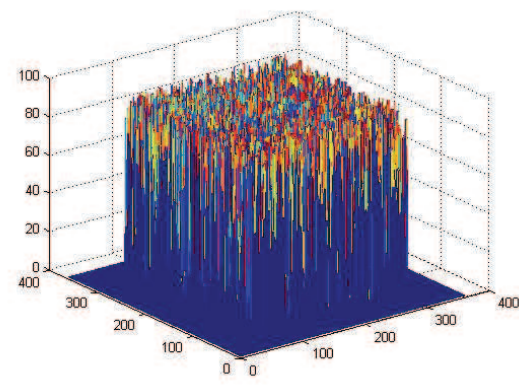

(b) Gaussian Noise

Figure 7. Depth maps using SML for simulated cone images

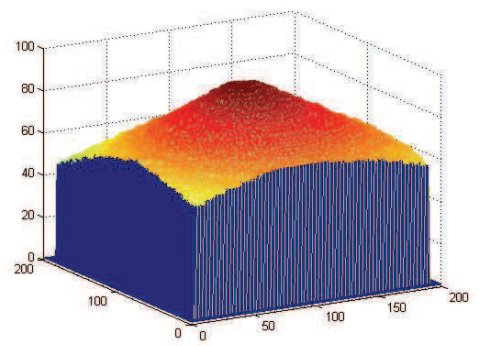

(a) No Noise

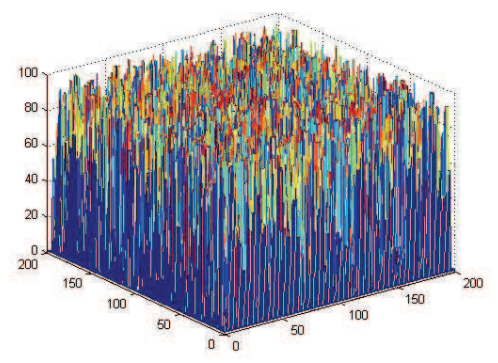

(b) Shot Noise

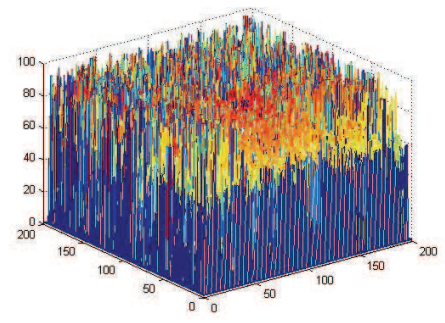

(c) Speckle Noise

Figure 8. Depth maps using SML for real cone images (Shot and Speckle Noise)

\section{Robust Focus Measure}

Based on the results shown in section 5, a robust focus measure is required that can perform well even in the presence of noise. In this section, a robust focus measure is presented for estimation of depth map. That depth map can further be used in techniques and algorithms leading to recovery of three dimensional structure of the object which is required in many high level vision applications. The focus measure presented here has shown robustness in the presence of noise as compared to the earlier focus measures. This new focus measure is 
based on an optical transfer function implemented in the Fourier domain. The results of the proposed focus measure have shown drastic improvement in estimation of depth map, with respect to the earlier focus measures, in the presence of various types of noise including Gaussian, Shot and Speckle noise. The focus measure is based on bipolar incoherent image processing and we call it Optical Focus Measure and denote it as FMo. (Poon and Banerjee, 2001) has discussed bipolar incoherent image processing in detail. Let $g(x, y)$ be input image frames, F \& F-1 be Fourier and inverse Fourier transform, $\mathrm{k}_{\mathrm{x}}$ and $\mathrm{k}_{\mathrm{y}}$ be spatial frequencies, $\sigma_{1}$ and $\sigma_{2}$ be filtering parameters, then mathematically, this focus measure is represented as:

$$
\operatorname{FM}_{\mathrm{O}}(\mathrm{i}, \mathrm{j})=\sum_{x=i-N}^{i+N} \sum_{y=j-N}^{j+N} \operatorname{Real}\left[\mathrm{F}^{-1}\left\{S\left(k_{x}, k_{y}\right) H\left(k_{x}, k_{y}\right)\right\}\right]
$$

where: $\mathrm{S}\left(\mathrm{k}_{\mathrm{x}}, \mathrm{k}_{\mathrm{y}}\right)=F|g(x, y)|^{2}, H\left(k_{x}, k_{y}\right)=\exp \left\{-\sigma_{1}\left(k_{x}^{2}+k_{y}^{2}\right)\right\}-\exp \left\{-\sigma_{2}\left(k_{x}^{2}+k_{y}^{2}\right)\right\}$

Hence, this focus measure becomes a filtering operation that provides the sharpness at pixel points in an image. The filtering operation depends upon $\sigma_{1}$ and $\sigma_{2}$. These values are adjusted to provide sharp focus measure even in the presence of noise. The operator responds to the high and medium frequency variations in the image intensity. The high and the medium frequency component of an image area is determined by processing in the Fourier domain and analyzing the frequency distribution. The processing in the frequency domain is particularly useful for noise reduction as the noise frequencies are easily filtered out. Figure 9 shows the filter with $\sigma_{1}=0.01$ and $\sigma_{2}=0.1$.

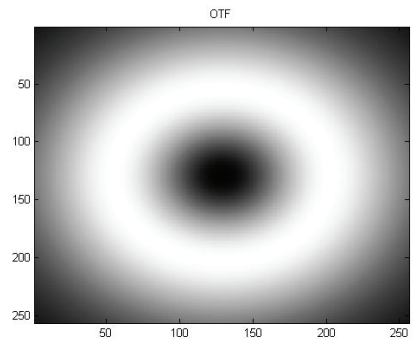

Figure 9. Filter designed with $\sigma_{1}=0.01$ and $\sigma_{2}=0.1$

This focus measure is applied on a sequence of 97 simulated cone images, 97 real cone images and 87 slanted planar object images. The resolution of the images is $360 \times 360$ pixels for both the simulated and real cone images and it is 200x200 pixels for the planar object. The results are compared with Sum of Modified Laplacian (SML), Gray Level Variance (GLV) method, Tenenbaum and $\mathrm{M}_{2}$ focus measures.

Consider Figure 10. Noise is added to the sequence of the images of simulated cone. Noise added is Gaussian with zero mean and variance equal to 0.05 . Figures 10(a) to 10(e) show the depth maps calculated using Tenenbaum, SML, GLV, $\mathrm{M}_{2}$ and $\mathrm{FM}_{\mathrm{O}}$. As can be seen from the figures, the 3D depth map obtained using $\mathrm{FM}_{\mathrm{O}}$ is clearly recognizable but that of $\mathrm{SML}$ and $\mathrm{M}_{2}$ have degraded significantly. Infact, the noise added to the pixel values is enhanced in the depth map for SML and $\mathrm{M}_{2}$ and hence it results in spikes originating from pixels all over the image. On the other hand, the result for $\mathrm{FM}_{\mathrm{O}}$ has also degraded but that degradation is very minor and various approximation techniques can still use this depth map to refine the result. Further, the results for Tenenbaum and GLV in Figures 10(a) \& (c) have also degraded with spikes on one side of the image. However, the central part of the cone is still recognizable. 


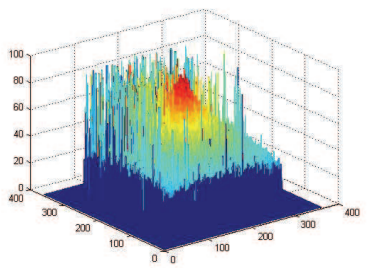

(a) Tenenbaum

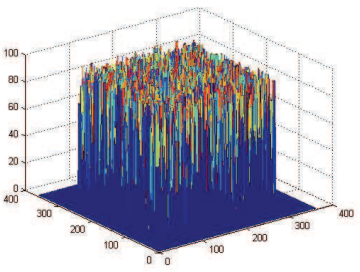

(b) SML

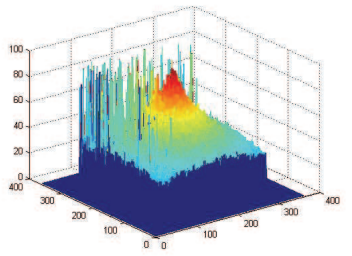

(c) GLV

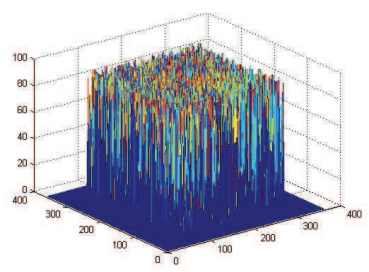

(d) $\mathrm{M}_{2}$

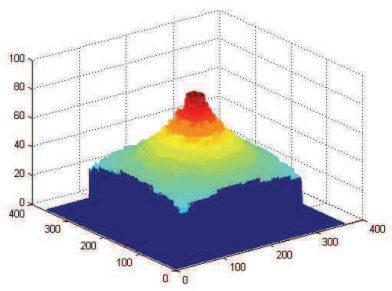

(e) $\mathrm{FM}_{\mathrm{O}}$

Figure 10. Depth maps for the simulated cone object when Gaussian noise is added

We used two metrics to compare these focus measures; Root Mean Square Error (RMSE) and Correlation. After comparing the results of RMSE of above mentioned focus measures, we found that the RMSE values are lowest for $\mathrm{FM}_{\mathrm{O}}$ in almost all the cases. Also, we found that $\mathrm{FM}_{\mathrm{O}}$ is highly correlated with the reference image and the correlation coefficient of $\mathrm{FM}_{\mathrm{O}}$ is highest among almost all the five focus measures. The reference image for comparison is the ground truth depth map for the simulated cone.

Consider tables 1 and 2 which show the results of the five focus measures in the presence of Gaussian noise. Table 1 is for RMSE and table 2 is for correlation result. From these tables, it is quite clear that the RMSE values are lowest for $\mathrm{FM}_{\mathrm{O}}$ and correlation is highest for $\mathrm{FM}_{\mathrm{O}}$. The result is shown for data with Gaussian noise of zero mean and varying variance values, i.e., variance $=0.5,0.05,0.005,0.0005,0.00005$. The performance of SML and $\mathrm{M}_{2}$ is the worst in this case till noise variance level of 0.0005 . GLV and Tenenbaum performance degrades for upper two noise levels while their performance increases considerably for the lower three noise levels. On the other hand, performance of $\mathrm{FM}_{\mathrm{O}}$ is almost constant for all noise levels. Figure 11 depicts this clearly. Similar results were observed for shot and speckle noise too.

\begin{tabular}{|c|c|c|c|c|c|}
\hline Variance & Tenenbaum & SML & GLV & $\mathrm{M}_{2}$ & FMo \\
\hline 0.5 & 28.5273 & 29.4557 & 28.3896 & 29.2447 & 15.1542 \\
\hline 0.05 & 20.4283 & 29.2709 & 20.0483 & 27.9341 & 14.2388 \\
\hline 0.005 & 19.639 & 20.8646 & 19.6283 & 19.6486 & 14.1992 \\
\hline 0.0005 & 19.6663 & 19.6249 & 19.6631 & 19.6258 & 14.2148 \\
\hline 0.00005 & 19.6779 & 19.6329 & 19.6682 & 19.6245 & 14.2117 \\
\hline No Noise & 19.7017 & 19.6557 & 19.6816 & 19.6535 & 14.2114 \\
\hline
\end{tabular}

Table 1. RMSE for Simulated Cone (Gaussian Noise) 


\begin{tabular}{|c|c|c|c|c|c|}
\hline Variance & Tenenbaum & SML & GLV & $\mathrm{M}_{2}$ & FMo \\
\hline 0.5 & 0.6138 & 0.5897 & 0.6165 & 0.5965 & 0.8707 \\
\hline 0.05 & 0.8699 & 0.5976 & 0.8829 & 0.6347 & 0.9116 \\
\hline 0.005 & 0.8985 & 0.8520 & 0.8982 & 0.8974 & 0.9124 \\
\hline 0.0005 & 0.898 & 0.8982 & 0.8978 & 0.8980 & 0.9120 \\
\hline 0.00005 & 0.8973 & 0.8979 & 0.8972 & 0.8981 & 0.9120 \\
\hline No Noise & 0.8991 & 0.8999 & 0.8985 & 0.9005 & 0.9119 \\
\hline
\end{tabular}

Table 2. Correlation for Simulated Cone (Gaussian Noise)

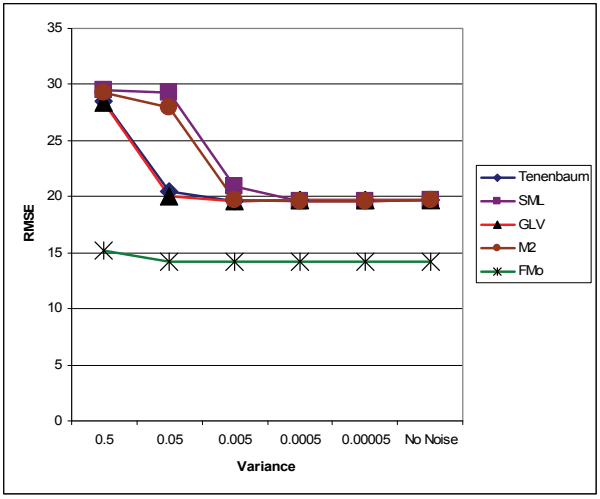

(a) RMSE

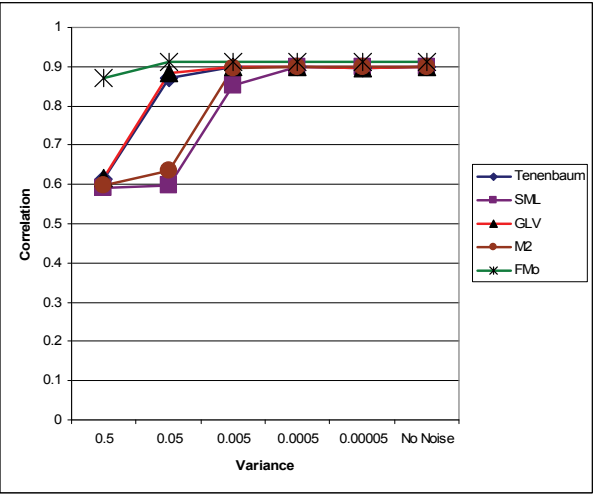

(b) Correlation

Figure 11. Comparison of Focus Measures (Gaussian Noise)

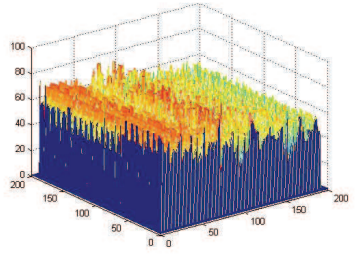

(a) Tenenbaum

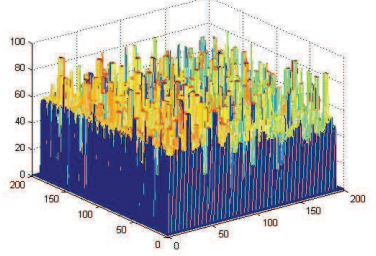

(b) SML

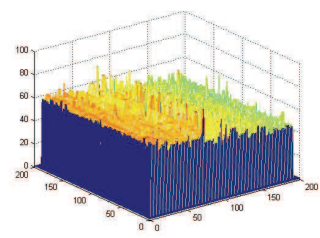

(c) GLV

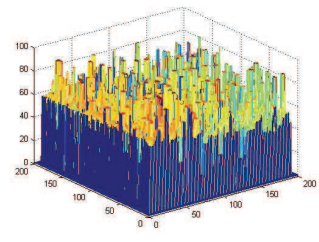

(d) $\mathrm{M}_{2}$

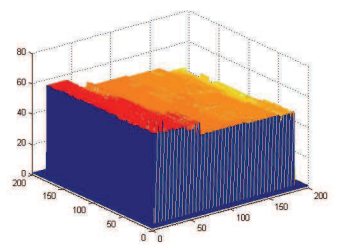

(e) $\mathrm{FMO}_{\mathrm{O}}$

Figure 12. Depth maps for the planar object when shot noise is added to the images

Now we add the shot noise to the sequence of images of the planar object. Consider figure 12 where figures 12 (a) to (e) show the depth maps for Tenenbaum, SML, GLV, $\mathrm{M}_{2}$ and $\mathrm{FM}_{\mathrm{O}}$, when the bipolar shot noise is added to the planar sequence of images. The noise density 
used is 0.0005 . As can be seen from the images, the depth maps for SML and $\mathrm{M}_{2}$ are again degraded with spikes originating from the pixels all over the image hence making the shape of the planar object unrecognizable. Further it cannot be used for more processing by any approximation techniques since the initial estimate is not good enough. However, it can also be seen from the depth maps that the result of Tenenbaum and GLV is better than SML and $\mathrm{M}_{2}$. On the other hand, consider figure 12(e). It shows the depth map calculated using the proposed focus measure FMo. Although there are few steps in the depth map but still the result is very good. Hence, again $\mathrm{FM}_{\mathrm{O}}$ performs better than rest of the focus measures. Similar results were observed for speckle noise too.

Keeping in view the results of all the objects, the following evaluations are made (Malik \& Choi, 2008):

- High noise level:

- Performance of all focus measures is affected in the presence of all noise types.

- Overall performance: FMo is the best followed by GLV \& Tenenbaum, and then SML \& $\mathrm{M}_{2}$.

- Low noise level:

- Gaussian noise: It affects the performance of SML and $\mathrm{M}_{2}$ but rest of the focus measures are not influenced.

- Shot noise: It affects all the focus measures except FMO $_{\mathrm{O}}$.

- Speckle noise: Focus measures are not affected by speckle noise.

- Overall performance: FMo is the best followed by GLV \& Tenenbaum, and then SML and $\mathrm{M}_{2}$.

- At medium noise levels:

- Performance of all focus measures is affected in the presence of all noise types.

- Gaussian noise: Performance of $\mathrm{FM}_{\mathrm{O}}, \mathrm{GLV}$ and Tenenbaum is comparable followed by SML \& $\mathrm{M}_{2}$.

- Shot noise: $\mathrm{FM}_{\mathrm{O}}$ outperforms other focus measures followed by Tenenbaum \& GLV, and then SML and $\mathrm{M}_{2}$.

- Speckle noise: $\mathrm{FM}_{\mathrm{O}}$ outperforms other focus measures followed by GLV \& Tenenbaum, and then SML and $\mathrm{M}_{2}$.

- Overall performance: $\mathrm{FM}_{\mathrm{O}}$ is the best followed by GLV \& Tenenbaum, and then SML and $\mathrm{M}_{2}$.

- Overall Performance:

- Gaussian Noise:

- $\quad \mathrm{FM}_{\mathrm{O}}$ outperforms at high and low noise levels and is comparable at medium noise level

- GLV and Tenenbaum show good performance too

- $\quad$ SML and $\mathrm{M}_{2}$ should be avoided except for low noise levels

- Shot Noise:

- $\quad \mathrm{FMO}_{\mathrm{O}}$ outperforms at all noise levels

- Rest of the focus measures should be avoided in the presence of shot noise

- $\quad$ Speckle Noise:

- $\quad \mathrm{FM}_{\mathrm{O}}$ outperforms at all noise levels

- GLV and Tenenbaum exhibits better performance

- $\quad \mathrm{SML}$ and $\mathrm{M}_{2}$ should be avoided except for low noise levels 


\section{Conclusion}

In this chapter, we considered the effects of illumination and window sizes on the focus measures for accurate calculation of depth map. We showed that the illumination effects can directly result in incorrect estimation of depth map if proper window size is not used for computation. We used two well established focus measures, i.e., Sum of Modified Laplacian and Gray Level Variance. We proved that larger window size results in two major errors. One is the introduction of blurring which results in smoothing of the object hence giving false impression of 3D smoothing in depth map. Second is the wrong extraction of frame numbers for depth map corresponding to the sharpest pixel values in the sequence of the images. Hence, it is suggested that smaller window size should be used with the upper bound of $5 \times 5$ on the size of the window. Hence, without pre-processing for image enhancement and without use of proper window size, it is not possible to obtain the accurate depth map for 3D shape recovery. It is worth noting that the problem defined in this chapter is not limited to Shape From Focus only. Rather most of the image processing techniques (especially 3D image recovery algorithms) based on window processing are marred with this problem, i.e., usage of large window size. Hence, this chapter provides guidance for research in this direction too.

In addition, we have presented a focus measure based on robustness in the presence of noise. We tested and compared this focus measure using simulated cone images, real cone images and slanted planar object images. The results show that this focus measure tends to perform better than the traditional focus measures when the noise is present in the images. We have shown the performance of various focus measures with three different types of noise, i.e., Gaussian, Shot and Speckle noise. The various focus measures used for comparison include Sum of Modified Laplacian (SML), Gray Level Variance (GLV), Tenenbaum and $\mathrm{M}_{2}$ focus measures which clearly indicate that the optical focus measure is equally good for images without noise and at the same time, it shows much enhanced performance in comparison to others in the presence of noise. It can be argued that some noise removal filter can be used before processing with the focus measure. However, as shown, the result of the proposed focus measure $\left(\mathrm{FM}_{\mathrm{O}}\right)$ is better even in the absence of noise. Further, FMO $_{\mathrm{O}}$ does not require noise removal filter because noise removal property is inherent within this technique. Lastly, we know that different types of noise removal filter are employed for different types of noise, e.g., median filter for shot noise, Weiner filter for Gaussian noise etc. Hence, some knowledge of noise is required before hand for the application of such filters. We used RMSE and Correlation metric measures to compare the performance of the earlier focus measures with our optical focus measure. The results clearly indicate that the RMSE values are lowest while the correlation values are the highest for the presented focus measure when compared with the SML, GLV, Tenenbaum and $\mathrm{M}_{2}$ focus measures at almost all the noise levels for all objects. It is concluded from the results that the best performance is shown by $\mathrm{FM}_{\mathrm{O}}$ followed by GLV, Tenenbaum, $\mathrm{M}_{2}$ and SML.

\section{Acknowledgements}

This work was supported by the Korea Science and Engineering Foundation (KOSEF) grant funded by the Korean government (MOST) (No. R01-2007-000-20227-0). The authors also acknowledge the support of Dr Asifullah Khan during the review of this chapter. 


\section{References}

Krotkov, E.P. Focusing, International Journal of Computer Vision, (1987) pp. 223-237.

Helmli, F.S. \& Scherer, S. (2001). Adaptive shape from focus with an error estimation in light microscopy, 2nd International Symposium on Image and Signal Processing and Analysis (ISPA01), pp. 188-193, Pula, Croatia

Nayar, S.K. \& Nakagawa, Y. Shape from focus, IEEE Transactions on Pattern Analysis and Machine Intelligence, Vol. 16, No. 8 (August 1994) pp. 824-831

Subbarao, M., Choi, T.-S. \& Nikzad, A. Focusing techniques, Optical Engineering, Vol. 32, No. 11 (November 1993) pp. 2824-2836

Malik, A.S. \& Choi, T.-S. Application of passive techniques for three dimensional cameras, IEEE Transactions on Consumer Electronics, Vol. 53, No. 2 (May 2007) pp. 258-264

Subbarao, M. \& Choi, T.-S. Accurate recovery of three dimensional shape from image focus, IEEE Transactions on Pattern Analysis and Machine Intelligence, Vol. 17, No. 3, (March 1995) pp. 266-274

Choi, T.-S., Asif, M. \& Yun, J. (1999). Three-dimensional shape recovery from focused image surface, IEEE International Conference on Acoustics, Signal and Speech Processing, Vol. 6, pp. 3269-3272, Arizona, US

Asif, M. \& Choi, T.-S. Shape from focus using multilayer feedforward neural network, IEEE Transactions on Image Processing, Vol. 10, No. 11 (November 2001) pp. 1670-1675

Ahmad, M.B. \& Choi T.-S. A Heuristic approach for finding best focused shape, IEEE Transactions on Circuits and Systems for Video Technology, Vol. 15, No. 4 (April 2005) pp. 566-574

Malik, A.S. \& Choi, T.-S. Consideration of illumination effects and optimization of window size for accurate calculation of depth map for 3D shape recovery, Pattern Recognition, Vol. 40, No. 1, (January 2007) pp. 154-170

Poon, T.-C. \& Banerjee, P. (2001). Contemporary optical image processing, 1st ed., Elsevier Science Ltd., New York

Malik, A.S. \& Choi, T.-S. A Novel Algorithm for Estimation of Depth Map using Image Focus for 3D Shape Recovery in the Presence of Noise, Pattern Recognition, Vol. 41, No. 7, (July 2008) pp. 2200-2225 


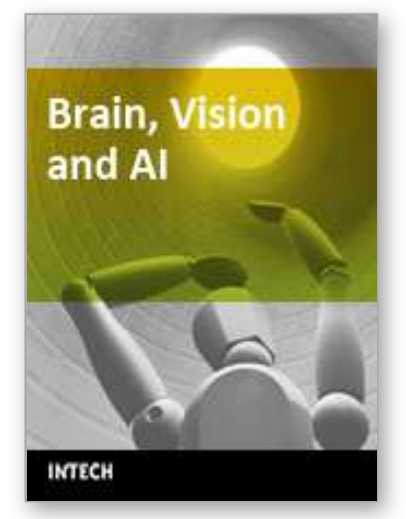

\author{
Brain, Vision and Al \\ Edited by Cesare Rossi
}

ISBN 978-953-7619-04-6

Hard cover, 284 pages

Publisher InTech

Published online 01, August, 2008

Published in print edition August, 2008

The aim of this book is to provide new ideas, original results and practical experiences regarding service robotics. This book provides only a small example of this research activity, but it covers a great deal of what has been done in the field recently. Furthermore, it works as a valuable resource for researchers interested in this field.

\title{
How to reference
}

In order to correctly reference this scholarly work, feel free to copy and paste the following:

Aamir Saeed Malik and Tae-Sun Choi (2008). Consideration of Various Noise Types and Illumination Effects for 3D Shape Recovery, Brain, Vision and AI, Cesare Rossi (Ed.), ISBN: 978-953-7619-04-6, InTech, Available from:

http://www.intechopen.com/books/brain_vision_and_ai/consideration_of_various_noise_types_and_illuminatio n_effects_for_3d_shape_recovery

\section{INTECH}

open science | open minds

\section{InTech Europe}

University Campus STeP Ri

Slavka Krautzeka 83/A

51000 Rijeka, Croatia

Phone: +385 (51) 770447

Fax: +385 (51) 686166

www.intechopen.com

\section{InTech China}

Unit 405, Office Block, Hotel Equatorial Shanghai

No.65, Yan An Road (West), Shanghai, 200040, China 中国上海市延安西路65号上海国际贵都大饭店办公楼 405 单元

Phone: +86-21-62489820

Fax: $+86-21-62489821$ 
(C) 2008 The Author(s). Licensee IntechOpen. This chapter is distributed under the terms of the Creative Commons Attribution-NonCommercialShareAlike-3.0 License, which permits use, distribution and reproduction for non-commercial purposes, provided the original is properly cited and derivative works building on this content are distributed under the same license. 